BMJ Open

Sport \&

Exercise

Medicine

\title{
The impact of 12 weeks walking football on health and fitness in males over 50 years of age
}

\author{
Josh Timothy Arnold, ${ }^{1}$ Stewart Bruce-Low, ${ }^{1}$ Luke Sammut ${ }^{2}$
}

To cite: Arnold JT, BruceLow S, Sammut L. The impact of 12 weeks walking football on health and fitness in males over 50 years of age. BMJ Open Sport Exerc Med 2015;1:e000048. doi:10.1136/bmjsem-2015000048

- Prepublication history for this paper is available online. To view these files please visit the journal online (http://dx.doi.org/10.1136/ bmjsem-2015-000048).

Accepted 21 September 2015

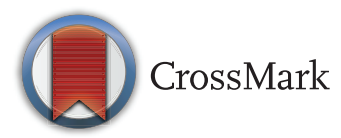

${ }^{1}$ School of Sport, Health and Social Sciences,

Southampton Solent University, Southampton, Hampshire, UK

${ }^{2}$ University Hospital

Southampton NHS

Foundation Trust,

Southampton, Hampshire, UK

Correspondence to

Josh Arnold;

josh.t.arnold@gmail.com

\section{ABSTRACT}

Aim: To describe and characterise anthropometrical and fitness changes following a 12-week walking football programme in individuals over the age of 50 years.

Methods: Following ethical approval, 10 male participants (mean (SD): age 66 (7) years) with a range of comorbidities completed a 12-week walking football programme, consisting of a single $2 \mathrm{~h}$ training session each week. Body mass, fat mass, fat free mass, maximal oxygen consumption, maximal heart rate, exercise time to exhaustion and isometric handgrip strength, were assessed at baseline and immediately following the intervention. Week-0-12 intervention differences were determined using means (95\% Cls) and t tests; effect sizes were calculated using Cohen's d (0.2 small, 0.5 medium, 0.8 large).

Results: 12 weeks walking football significantly reduced body fat mass (week 0, 27.4 (9.0) $\mathrm{kg}$ versus week 12, $24.4(8.9) \mathrm{kg}, \mathrm{p}=<0.05, \mathrm{~d}=1.0)$ and reduced percentage body fat (week $0,30.3(8.2) \%$ versus week 12, $27.5(8.5) \%, p=<0.05, d=1.0)$. A significant increase in time to volitional exhaustion during increamental exercise (week 0, 545 (102) s versus week 12, 603 (102) s, $p=<0.05, d=0.7$ ) was observed without any change in peak blood lactate. Nonsignificant differences with medium effect sizes were seen for a reduction whole body mass, increase in lean body mass and a reduction in body mass index.

Conclusions: This investigation suggests the potential efficacy of walking football as a public health intervention, even in populations presenting a range of comorbidities, with future research investigating its move to scale.

\section{INTRODUCTION}

Walking football has recently emerged as a popular variant of association football, targeting individuals over the age of 50 years. Though walking football adheres to the general rules of football, it does not allow players to run (as defined by a loss of contact of both feet with the ground) or side-tackle during open play. ${ }^{1}$ A well-advertised objective of walking football is to keep individuals over the age of 50 involved in sport. Indeed,

\section{What are the new findings?}

- Twelve weeks walking football, in the form of one $2 \mathrm{~h}$ training session per week, positively altered a range of anthropometrical and fitness parameters.

- A significant reduction was seen in body fat mass and percentage body fat between 0 and 12 weeks.

- A significant increase was seen in time to volitional exhaustion during increamental exercise, without any change in peak blood lactate between 0 and 12 weeks.

- Walking football is safe and efficacious, not only for healthy individuals but also those with various exercise limiting comorbidities aged over 50 .

individuals aged 45-55 currently represent the single highest prevalence of primary diagnoses of obesity; furthermore the number of primary diagnoses of obesity continues to rise in those aged 65 and over, despite promising plateaus in other age groups. ${ }^{2}$

Walking football has been increasingly referred to in the media in relation to its positive association with physical activity and subsequently health and well-being. Indeed, physical activity and exercise reduce all-cause mortality and other health-related disorders $^{3} 4$ in a dose-response fashion. ${ }^{5}$ However, while the capacity of walking football as a means to enhance health and fitness in populations over 50 years is likely, as yet little empirical evidence exists to consolidate this.

To date, the physiological benefit of association football have been well documented ${ }^{6}$ however such information has yet to be outlined relative to walking football. Specifically, because running is forbidden, the absolute physiological intensity and energy cost of the sport is lower than in association football. The relative physiological requirements of 
the two variants of the game are possibly more comparable when played by their specific target populations. Indeed, the comparative aerobic demand of walking is known to increase with age. ${ }^{78}$ Thus, walking football may provide a sufficient physiological stimulus to augment aerobic fitness in older populations in addition to other anthropometrical and health adaptations. Effective and safe methods of improving aerobic fitness are important to public health agendas.

This study therefore aimed to characterise and describe any physiological and anthropometrical changes exhibited following a 12-week walking football programme as completed by older age populations ( $>50$ years). It was hypothesised that participation in 12 weeks of regular walking football would significantly enhance various physiological markers of health and fitness in individuals over 50 years of age.

\section{METHODS}

\section{Participants}

Ten male participants (mean (SD): age 66 (7) years, height $1.77(0.07) \mathrm{m}$, body mass $89.2(9.1) \mathrm{kg}$ ) with a range of comorbidities (table 1) were recruited using convenience-sampling methods from a local walking football club in the Southampton area between January and February 2015. Inclusion criteria detailed; otherwise healthy males aged over 50 years, not currently partaking in any other exercise or health-related programme or within the previous 6 months. Where possible, priority was granted to the newest players within the club, in order to best assess the potential impact of walking football on the health and fitness of individuals considering joining. All participants provided written informed consent. Ethical approval was granted by the Ethics Committee of the School of Sport, Health and Social Sciences at Southampton Solent University.

\section{Design and intervention}

The study utilised a repeated measures, pre-post intervention design, following a 1-month gap in regular training. All participants reported to the sport science laboratories at Southampton Solent University to complete baseline testing. Tests included; anthropometrical assessment, incremental exercise to volitional exhaustion,

Table 1 Comorbidities presented by a sample of 10 male walking football players over the age of 50

\begin{tabular}{ll}
\hline Co-morbidity & Frequency \\
\hline Hypertension & 4 \\
Knee osteoarthritis & 3 \\
Type 2 diabetes mellitus & 2 \\
Spinal stenosis & 1 \\
Atrial fibrillation & 1 \\
Bronchitis & 1 \\
Other comorbidities & 4 \\
\hline
\end{tabular}

grip strength assessment and various health measures. One week later, all participants began the 12-week walking football programme. Throughout the 12 weeks individuals attended a single $2 \mathrm{~h}$ training session each week as usual. Sessions comprised of multiple short 5-a-side games (15-20 min), semistructured in nature. At 12 weeks, all participants underwent testing once more at the Southampton Solent University. Testing at 12 weeks was undertaken at the same time, and in an identical manner to baseline testing, to minimise the effects of diurnal rhythms.

\section{Physiological procedures}

One week before testing, participants were fully briefed with regards to the study aims and design. Furthermore, $24 \mathrm{~h}$ prior to testing participants received a standardised message reminding them to avoid any strenuous exercise, and to refrain from any consumption of alcohol and caffeine. Participants were also encouraged to maintain regular eating habits and drink ad-lib in order to keep well hydrated. The following morning, participants reported to the sport science laboratories with their usual exercise clothing. Participants were asked to complete a physical activity readiness questionnaire (PAR-Q) in order to highlight any existing medical conditions, before undergoing various health checks and demographic measures including; mass via balance scales (Seca Flat Scales 710, Seca, UK), stature via stadiometer (Harpenden stadiometer, Holtain Ltd, UK), blood pressure via automated sphygmomanometer (Boso Medicus Automated Sphygmomanometer, Bosch and Sochn Germany, Jungingen, Germany), body composition via air displacement method (Bod Pod Gold Standard, Life Measurement Inc, USA), hydration status via portable osmometer (Osmocheck Pocket, Vitech Scientific Limited, UK), blood glucose via fingertip capillary sampling (Biosen C Line, EKF Diagnostic, Germany) and finally a 12-lead non-medical diagnostic ECG (Oxycon Mobile, Jaeger, Germany). In order to participate within any further exercise testing participants were required to present euhydration $(<700 \mathrm{mOsm} / \mathrm{kg}),{ }^{9}$ a stable blood glucose value (within $4.4-6.1 \mathrm{mmol} / \mathrm{L}$ ), ${ }^{10}$ a stable resting blood pressure $(<160 / 90 \mathrm{~mm} \mathrm{Hg}$ systolic/diastolic), ${ }^{11}$ and a regular ECG trace.

Following prechecks, participants first completed an isometric hand grip strength test using a digital 5101 Grip-D handgrip dynamometer (Takei, Japan). Isometric grip strength was taken as the maximum value from three maximal voluntary isometric contractions. Hand position was controlled by keeping the dynamometer handle setting standardised across trials, as selected by the participant, and the participants arm adducted at their side with elbows at $90^{\circ}$, as recommended by the American Society of Hand Therapists. ${ }^{12}$ Participants were instructed to squeeze the handle of the dynamometer progressively harder, culminating in a maximal voluntary contraction after $3 \mathrm{~s}$. 
Following isometric grip strength, participants completed an incremental exercise test to volitional exhaustion on a motorised treadmill (ELG 70/200, Woodway, Germany) using a Bruce protocol. ${ }^{13}$ Where necessary, participants were familiarised with the treadmill, before conducting a self-paced warm up for 5 min prior to exercise. The protocol was then started. The treadmill was initiated at $2.74 \mathrm{~km} / \mathrm{h}$ at a $10 \%$ gradient, after which the speed and gradient of the treadmill were incrementally increased every $3 \mathrm{~min}$ until volitional exhaustion. Oxygen uptake $\left(\mathrm{V}_{2}\right)$ and respiratory exchange ratio (RER) were continually assessed throughout the test via a breath-by-breath on-Line gas analysis system (Oxycon Pro, Jaeger, Germany). In addition, heart rate via remote transmitter (RCX5, Polar Electro, Finland) and rate of perceived exertion via Borg 6-20 scale $^{14}$ were recorded $15 \mathrm{~s}$ prior to the end of each stage and at exhaustion. Verbal encouragement was provided once RPE exceeded 15. At exhaustion blood lactate via fingertip capillary blood sampling (Lactate Pro, Ark Ray Inc, Kyoto, Japan) was assessed. To ascertain whether or not a maximal effort was achieved during the test, the following criteria were considered; a plateau in $\dot{\mathrm{V}} \mathrm{O}_{2}$, RER $>1.15$, heart rate within 10 beats of maximal (age predicted), RPE $>19$, and a blood lactate value of $>8 \mathrm{mmol} / \mathrm{L}^{15}$

\section{Data analysis}

Data are presented as means (SD) or $(95 \%$ CI). Inferential statistical analysis was conducted using the software package SPSS (V.20, IBM, Portsmouth, UK). Statistical significance was set at $p \leq 0.05$. To evaluate the statistical significance of the training intervention paired samples $\mathrm{t}$ tests were used to assess differences between week-0 and week-12 measurements for each parameter. The magnitude of change between week-0 and week-12 was calculated using Cohen's d. Finally, to investigate any trends in response to the intervention, week- 0 values were correlated (Pearson's r) against the change in values for each parameter (week-0 to week-12), in addition to age.

\section{RESULTS}

All particpants completed the 12-week intervention. Mean values and for all anthropometrical and exercise parameters are displayed in table 2.

\section{Resting parameters}

Twelve weeks of walking football positively altered all anthropometrical parameters. A statistically significant $11 \%$ reduction was observed in body fat mass (week 0 , 27.4 (9.0) $\mathrm{kg}$ versus week 12, 24.4 (8.9) $\mathrm{kg}, \mathrm{p}=<0.05$, $\mathrm{d}=1.0$ ) and a $9 \%$ reduction was observed in percentage body fat (week $0,30.3(8.2) \%$ versus week 12, 27.5 (8.5) $\%, p=<0.05, d=1.0)$. Non-statistically significant differences, however with medium effect sizes, were observed in other anthropometrical variables; a $2 \%$ reduction in whole body mass (week $0,89.2(9.1) \mathrm{kg}$ versus week 12, $87.4(8.56) \mathrm{kg}, \mathrm{p}=0.08, \mathrm{~d}=0.6)$; a $4 \%$ increase in lean body mass (week 0,61.8 (6.0) $\mathrm{kg}$ versus week 12, $64.3(8.0) \mathrm{kg}, \mathrm{p}=0.06, \mathrm{~d}=0.7)$; and a $3 \%$ reduction in body mass index (BMI) (week 0, 29 (3) versus week 12, $28(4), \mathrm{p}=0.06, \mathrm{~d}=-0.7)$. No correlations were observed between week- 0 measurements and the difference in week-0 to week-12 measurements for each anthropometrical parameter. In addition no correlation was observed between age and the difference in week- 0 to week-12 measurements for each parameter.

Systolic blood pressure increased by $4 \%$ after the 12-week walking football programme (week 0, 149 (14) versus week 12, $155(10), \mathrm{p}=0.01, \mathrm{~d}=1.0)$. However, 2 of the 10 participants reported (informally) that they had complied poorly with their antihypertensive medication during the study period. Furthermore, a significant correlation was observed between week-0 systolic blood

Table 2 Anthropometrical and fitness parameters assessed before and after a 12-week walking football intervention for males over 50 years old

\begin{tabular}{|c|c|c|c|c|c|}
\hline & Week 0 & Week 12 & Mean difference (W12-W0) & p Value & ES \\
\hline Mass $(\mathrm{kg})$ & $89.2(9.1)$ & $87.4(8.56)$ & $-1.8(-0.3$ to 3.9$)$ & 0.08 & -0.6 \\
\hline Fat mass $(\mathrm{kg})$ & $27.4(9.0)$ & $24.4(8.9)$ & $-3.0(0.8$ to 5.2$)$ & $0.01^{*}$ & -1.0 \\
\hline Lean mass (kg) & $61.8(6.0)$ & $64.3(8.0)$ & $2.5(-5.1$ to 0.2$)$ & 0.06 & 0.7 \\
\hline Body fat (\%) & $30.3(8.2)$ & $27.5(8.5)$ & $-2.8(0.8$ to 4.8$)$ & $0.01^{*}$ & -1.0 \\
\hline BMI & $29(3)$ & $28(4)$ & $-1(0$ to 1$)$ & 0.06 & -0.7 \\
\hline Systolic BP $(\mathrm{mm} \mathrm{Hg})$ & 149 (14) & $155(10)$ & $7(-11$ to -2$)$ & $0.01^{*}$ & 1.0 \\
\hline Diastolic BP $(\mathrm{mm} \mathrm{Hg})$ & $83(11)$ & $85(8)$ & $2(-8$ to 3.9$)$ & 0.4 & 0.3 \\
\hline$\dot{\mathrm{V}} \mathrm{O}_{2 \text { peak }}(\mathrm{mL} / \mathrm{kg} / \mathrm{min})$ & $28.6(6.2)$ & $30.0(6.8)$ & $1.4(-3.7$ to 1.0$)$ & 0.2 & 0.4 \\
\hline Peak heart rate (bpm) & $147(15)$ & $137(27)$ & $-9(-5$ to 22$)$ & 0.1 & -0.5 \\
\hline Peak blood lactate (mmol/L) & $7.2(3.1)$ & $8.6(3.5)$ & $1.4(-3.3$ to 0.4$)$ & 0.1 & 0.5 \\
\hline Time to exhaustion (s) & 545 (102) & $603(102)$ & $58(-114$ to 2$)$ & $0.04^{*}$ & 0.7 \\
\hline Grip strength $(\mathrm{kg})$ & $40(5)$ & $39(7)$ & $-1(-3$ to 5$)$ & 0.7 & -0.2 \\
\hline
\end{tabular}


pressure and the change in systolic blood pressure (week-0 to week-12) $(\mathrm{r}=-0.7, \mathrm{p}=<0.05)$, suggesting that those individuals with the lowest systolic blood pressure at the onset of the programme experienced the greatest magnitude of increase in systolic blood pressure. No change was reported in diastolic blood pressure.

\section{Exercise parameters}

The extent of the impact of 12 weeks walking football varied across exercise parameters. Despite no statistically significant difference in $\dot{\mathrm{V}}_{2 \text { peak, }}$ blood lactate, or peak heart rate, a statistically significant $11 \%$ increase was observed in time to volitional exhaustion during increamental exercise (week 0, 545 (102) s versus week 12, 603 (102) $\mathrm{s}, \mathrm{p}=<0.05, \mathrm{~d}=0.7)$. No correlations were observed between week- 0 measurements and the difference in week-0 to week-12 measurements for each exercise parameter. In addition no correlation was observed between age and the difference in week- 0 to week-12 measurements for each exercise parameter.

\section{DISCUSSION}

The 12-week walking football programme, in the form of one $2 \mathrm{~h}$ training session per week, positively altered a range of anthropometrical and fitness parameters. Our original data are consistent with a recent meta-analysis of walking-based randomised control trials, which reports an overall reduction in body mass $(1.8 \%)$, percentage body fat $(3.5 \%)$ and BMI $(2 \%)^{16}$ - key risk factors for cardiovascular disease.

Mixed findings were presented across other fitness and physiological parameters. Walking football significantly increased time to exhaustion at 12-weeks, with no change in peak blood lactate and a slight reduction in peak heart rate, suggesting enhanced exercise tolerance. Many individuals over the age of 50 have concomitant exercise-limiting comorbidities such as cardiovascu$\operatorname{lar}^{17}{ }^{18}$ and musculoskeletal disorders. ${ }^{19} \quad 20$ Aligning within similar interventions, ${ }^{18}$ walking football could therefore provide an appropriate activity to encourage such individuals to exercise at a lower intensity while improving tolerance to exercise. This may be important for the maintenance of functional independence in this population, subsequently improving their potential ability to perform activities of daily living and quality of life. Owing to illness and disability have major adverse effects on quality of life, improved physical function might be expected to cause parallel increases in quality of life. ${ }^{21}$ Furthermore the results of the present pilot study show that walking football is safe and effective, not only for healthy individuals but also those with various exercise limiting comorbidities aged over 50 as displayed in table 1.

In contrast, no significant change was reported in $\dot{\mathrm{V}} \mathrm{O}_{2 \text { peak }}$. Thus, while the programme enhanced exercise tolerance, it is possible that the intensity of the exercise programme was insufficient to significantly enhance aerobic fitness. However, when comparing the small 5\% increase in aerobic capacity observed here to the significant $10 \%$ increase in aerobic capacity as reported by Murtagh et $a l^{16}{ }^{16}$ it is more probable that the small effect may result from the low exercise volume, and less from the low exercise intensity. Though the jury is currently out regarding the effectiveness of low versus highintensity exercise for use in public health work, ${ }^{22}$ it would be perhaps sensible to first investigate an increase in training volume in this specific population group, to ensuring safety and prevent injury.

Contrasting other parameters, systolic blood pressure increased during the 12-week programme. This finding disagrees with previous knowledge that physical activity is one of the most effective non-pharmacological treatments for hypertension. Several studies have found significant reductions in resting blood pressure of hypertensive patients after physical activity. ${ }^{16}{ }^{22-25}$ This result may be due to poor adherence to antihypertensive medication, as reported by several participants. Previous literature suggested a 3-9 $\mathrm{mm} \mathrm{Hg}$ difference in blood pressure between compliant and non-compliant receivers of antihypertensive medication, ${ }^{26}$ similar to the magnitude of difference observed in the current study. Note this suggestion provides a level of speculation, as individuals who are on antihypertensive medication, may be able to achieve blood pressure control on lower doses of medication, or possibly without any medication with the instruction of physical activity. ${ }^{27}$ Consequently, work here should seek to provide education alongside walking football programmes creating a holistic approach. Other confounding influences may have included, 'white coat hypertension' and circadian blood pressure fluctuations.

\section{Limitations}

The current investigation offers exciting prospects for the use of walking football as a public health tool, however such findings must be considered against potential limitiations of the study. Foremost, future research is required to ascertain and the extent of such findings relative to an appropriate control group, and also the potential impact that the programme may exert on other nutritional and lifestyle habits. Such factors were not considered within this pilot work, however may be important given the potentially positive effect of walking football on attitudes towards healthy behaviour as highlighted through unpublished work undertaken by colleagues at Southampton Solent University.

\section{CONCLUSION}

This investigation provides evidence that 12 weeks walking football, in the form of one $2 \mathrm{~h}$ training session per week, positively altered anthropometrical and fitness parameters. This suggests the potential efficacy of walking football as a public health intervention, even in populations presenting a range of comorbidities. Future research should investigate its move to scale. 
Twitter Follow Josh Arnold at @Josh_T_Arnold

Acknowledgements The authors gratefully acknowledge Matthew York (Hampshire FA) and Ray Murphy (Eastleigh FC) for promoting the study and their help with participant recruitment.

Competing interests None declared.

Ethics approval Southampton Solent University.

Provenance and peer review Not commissioned; externally peer reviewed.

Open Access This is an Open Access article distributed in accordance with the Creative Commons Attribution Non Commercial (CC BY-NC 4.0) license, which permits others to distribute, remix, adapt, build upon this work noncommercially, and license their derivative works on different terms, provided the original work is properly cited and the use is non-commercial. See: http:// creativecommons.org/licenses/by-nc/4.0/

\section{REFERENCES}

1. Hampshire FA. Walking football HFA laws of the game. 2015. http:// www.hampshirefa.com/ /media/countysites/hampshirefa/ walking-football/hfa-walking-football-laws-of-the-game-updated-july2015.ashx (accessed 30 Jul 2015).

2. HSCIC. Statistics on obesity, physical activity and diet: England. 2014. http://www.hscic.gov.uk/catalogue/PUB13648/

Obes-phys-acti-diet-eng-2014-rep.pdf (accessed 30 Jul 2015).

3. Paffenbarger RS Jr, Hyde RT, Wing AL, et al. Physical activity, all-cause mortality, and longevity of college alumni. $N$ Engl $J$ Med 1986;314:605-13.

4. Nocon M, Hiemann T, Müller-Riemenschneider F, et al. Association of physical activity with all-cause and cardiovascular mortality: a systematic review and meta-analysis. Eur J Cardiovasc Prev Rehabil 2008;15:239-46.

5. Lee IM, Skerrett PJ. Physical activity and all-cause mortality: what is the dose-response relation? Med Sci Sports Exerc 2001;33: S459-71; discussion S493-4.

6. Bangsbo J, Junge A, Dvorak J, et al. Executive summary: football for health-prevention and treatment of non-communicable diseases across the lifespan through football. Scand J Med Sci Sports 2014;24(Suppl 1):147-50.

7. Waters RL, Hislop HJ, Perry J, et al. Comparative cost of walking in young and old adults. J Orthop Res 1983;1:73-6.

8. Martin PE, Rothstein DE, Larish DD. Effects of age and physical activity status on the speed-aerobic demand relationship of walking. J Appl Physiol 1992;73:200-6.
9. Manore M, Meyer NL, Thompson J. Sport nutrition for health and performance. Leeds: Human Kinetics, 2009.

10. Pooler C. Porth pathophysiology: concepts of altered health states. Philadelphia: Lippincott Williams \& Wilkins, 2009.

11. McArdle WD, Katch FI, Katch VL. Essentials of exercise physiology. Philadelphia: Lippincott Williams \& Wilkins, 2006.

12. Fess EE, Moran C. Clinical assessment recommendations. Indianapolis: American Society of Hand Therapists, 1981.

13. Bruce RA. Exercise testing of patients with coronary heart disease. Principles and normal standards for evaluation. Ann Clin Res 1971;3:323-32.

14. Borg GA. Psychophysical bases of perceived exertion. Med Sci Sports Exerc 1982;14:377-81.

15. British Association of Sport and Exercise Sciences. Guidelines for the physiological testing of atheletes. London: Routledge, 1997.

16. Murtagh EM, Nichols L, Mohammed MA, et al. The effect of walking on risk factors for cardiovascular disease: an updated systematic review and meta-analysis of randomised control trials. Prev Med 2015;72:34-43.

17. Mozaffarian D, Benjamin EJ, Go AS, et al. American Heart Association Statistics Committee and Stroke Statistics Subcommittee. Heart disease and stroke statistics. Circulation 2015;131:e29-322.

18. Gupta S, Elkins MR. Supervised walking training improves maximum and pain-free walking distances in people with intermittent claudication. Br J Sports Med 2014;48:1130-2.

19. Zhang Y, Jordan JM. Epidemiology of osteoarthritis. Clin Geriatr Med 2010;26:355-69.

20. Gheno R, Cepparo JM, Rosca CE, et al. Musculoskeletal disorders in the elderly. J Clin Imaging Sci 2012;2:39.

21. Geirsdottir OG, Arnarson A, Briem K, et al. Physical function predicts improvement in quality of life in elderly Icelanders after 12 weeks of resistance exercise. J Nutr Health Aging 2012;16:62-6.

22. Biddle SJ, Batterham AM. High-intensity interval exercise training for public health: a big HIT or shall we HIT it on the head? Int J Behav Nutr Phys Act 2015;12:95.

23. Dickinson HO, Mason JM, Nicolson DJ, et al. Lifestyle interventions to reduce raised blood pressure: a systematic review of randomized controlled trials. J Hypertens 2006;24:215-33.

24. Fagard $\mathrm{RH}$, Cornelissen VA. Effect of exercise on blood pressure control in hypertensive patients. Eur J Prev Cardiol 2007;14:12-17.

25. Cornelissen VA, Smart NA. Exercise training for blood pressure: a systematic review and meta-analysis. J Am Heart Assoc 2013;2: e004473.

26. Costa FV, D'Ausilio A, Bianchi C, et al. Adherence to antihypertensive medications. High Blood Press Cardiovasc Prev 2009;16:101-10.

27. Kokkinos PF, Papademetriou V. Exercise and hypertension. Coron Artery Dis 2000;11:99-102. 